\title{
The volume-outcome relationship in kidney cancer: is more really better?
}

\author{
Brian T. Kadow, Shreyas S. Joshi, Alexander Kutikov, Elizabeth Handorf, Marc C. Smaldone, \\ Robert G. Uzzo, Daniel M. Geynisman \\ Fox Chase Cancer Center, Philadelphia, PA, USA \\ Correspondence to: Daniel M. Geynisman. Fox Chase Cancer Center, 333 Cottman Ave., Philadelphia, PA 19111, USA. Email: daniel.geynisman@fccc.edu. \\ Provenance: This is an invited article commissioned by the Section Editor Dr. Xiao Li (Department of Urology, Jiangsu Cancer Hospital, Jiangsu \\ Institute of Cancer Research, Nanjing Medical University Affiliated Cancer Hospital, Nanjing, China). \\ Comment on: Hsu RCJ, Barclay M, Loughran MA, et al. Impact of hospital nephrectomy volume on intermediate- to long-term survival in renal cell \\ carcinoma. BJU Int 2019. [Epub ahead of print].
}

Submitted Sep 11, 2019. Accepted for publication Sep 18, 2019.

doi: 10.21037/atm.2019.09.108

View this article at: http://dx.doi.org/10.21037/atm.2019.09.108

Multiple studies have suggested a correlation between hospital surgical volume and clinical outcomes, including oncologic outcomes such as cancer-specific survival $(1,2)$. The fact that high-volume surgeons and institutions have superior outcomes may be related to improved technical surgical experience, streamlined perioperative protocols, access to multidisciplinary care, or the availability of new treatment modalities through clinical trials. This phenomenon has been reported in the literature across a range of disease states and surgical interventions (3-9). This volume-outcome relationship, which has been studied since the late 1970's, has led many to advocate for the regionalization of complex cancer surgery $(10,11)$. Many of these studies, however, have been limited in the length of follow-up and have only reported perioperative and shortterm (30 days) outcomes (12). However, more relevant cancer-related quality metrics might be evaluated through longer-term follow-up, such as 1- or 5-year cancer-specific survival or overall survival (OS).

Within the urologic literature, the effect of regionalization of care for renal cell carcinoma (RCC) has been studied in both patients with localized and metastatic disease. These studies suggest improved perioperative outcomes such as lower complication rates, fewer blood transfusions and shorter length of stay at high-volume centers, as well as improved survival outcomes for patients with metastatic disease receiving systemic therapy (13-15).

Adding to the volume-outcome literature for RCC, Hsu and colleagues recently published a retrospective administrative database study evaluating the relationship between a hospital's surgical volume for treatment of RCC and short, intermediate, and long-term overall mortality (16). They identified 12,912 adults with a diagnosis of clinically localized RCC who underwent surgical intervention between 2000 and 2010 within the National Health Service (NHS) system in the United Kingdom. Annual operative caseloads were calculated, and hospitals were categorized into low ( $<20$ cases), medium (20-39 cases), or high ( $\geq 40$ cases) volume centers based on their annual radical or partial nephrectomy surgical volume. Univariate and multivariate models were used to evaluate the relationship between surgical volume and short (30 days-1 year), intermediate (1-3 years), and long-term (3-5 years) overall survival. All patients had at least 5 years of post-operative follow-up. The authors found that patients undergoing treatment at high volume institutions had more comorbidities, more stage T1 tumors, and on multivariable analyses, had superior short-term (up to 1 year) outcomes, with a $34 \%$ reduction in mortality hazard (HR, 0.66 ; $95 \%$ CI $0.53-0.83$; $\mathrm{P}<0.01)$ over medium- or low-volume institutions. They calculated that 71 patients would need to be treated at a high-volume center to prevent 1 mortality during the first year.

Interestingly, however, the authors found that after 1 year of follow-up, there did not appear to be a survival benefit for patients treated at high volume centers. Given that the analysis of each time period was dependent on patients surviving the previous follow-up interval, once a patient 
passed through the initial post-operative "bottleneck", a center's clinical volume ceased to be a factor impacting their survival. Therefore, the differences in short-term (up to 30 days) mortality may be the main factor contributing to the superior mortality outcomes at high-volume institutions for up to 1 year. In subgroup analysis, the authors found attenuated, non-significant associations between hospital volume and mortality for patients with locally advanced (T3 or T4) disease, potentially suggesting that tumor biology may be a more important driver of outcomes in these patients.

This study by Hsu and colleagues is the first to demonstrate a survival benefit for surgical treatment of RCC at high volume centers beyond 30 days, as the previous literature has only evaluated perioperative and short-term outcomes. This study lends further credence to the view that regionalization of complex cancer care leads to superior clinically relevant outcomes. As the authors note, however, the exact factors that lead to improved outcomes at high-volume centers have not yet been elucidated, but they theorized it may be due to improved perioperative outcomes, standardization of perioperative pathways, availability of multidisciplinary care that is not available at lower volume centers, more rapid adoption of new clinical guidelines, or access to novel treatments through clinical trials. How to improve these factors at lower-volume centers is not entirely clear and warrants future study. For instance, assessment of outcomes of low-volume surgeons at high-volume centers, as has been done in prior work, could be informative (17).

The authors acknowledge their study limitations such as the low rate of complete TNM staging and the lack of knowledge of neoadjuvant or adjuvant systemic therapy, which could have significant implications for cancerspecific and overall survival. Another limitation of the study is the lack of RCC-specific mortality, particularly given the fact that the largest effect of hospital volume on mortality appeared to be in patients with T1 disease. In addition, the division of centers into low-, intermediate-, and high-volume is somewhat artificial. A continuous volume-outcome measure may be more robust. Another confounding finding in the study was that high-volume hospitals operated on a statistically higher rate of patients with T1 disease than lower volume centers, suggesting that high-volume hospitals in this study may have been less likely to offer active surveillance for small renal masses than their lower-volume counterparts. Furthermore, a higher rate of patients with $\mathrm{T} 1$ disease at high-volume centers could have an impact on impact mortality rates because these patients would be expected to have less complicated surgical procedures, lower intraoperative blood loss, and potentially shorter recovery times than in patients with higher stage ( $\mathrm{T} 2+)$ tumors.

Despite the study's strengths, how these findings should be applied to clinical practice is unclear. Although regionalization of the care of RCC (and other complex oncologic) patients may be beneficial, it is extremely challenging to accomplish from both logistic and financial perspectives. While the call for regionalization may be a reasonable endorsement within the NHS system that formed the basis for this study, such regionalization would likely be impossible within the complex American healthcare environment. Furthermore, some have raised concerns that regionalization may create significant barriers to access of care for the most vulnerable of patient populations (18). Rather, promoting a closer relationship and partnership between centers of excellence and community sites so as to share and disseminate practice patterns and insights, hosting joint tumor boards, creating user-friendly clinical pathways, and leveraging technology to help improve the care of patients in the community may be a more reasonable approach.

Finally, as newer targeted agents and immunotherapy strategies become increasingly effective and more frequently utilized in patients with higher stage disease in the perioperative period, or in those patients who eventually progress to metastatic disease, these therapies may subsequently "level the playing field" and potentially minimize some of the effect that hospital surgical volume appears to have on survival metrics, particularly in the longterm setting. In the meantime, providers at lower-volume centers should not hesitate to "phone a friend" on behalf of their patient.

\section{Acknowledgments}

None.

\section{Footnote}

Conflicts of Interest: The authors have no conflicts of interest to declare.

Ethical Statement: The authors are accountable for all aspects of the work in ensuring that questions related to the accuracy or integrity of any part of the work are 
appropriately investigated and resolved.

\section{References}

1. Birkmeyer JD, Sun Y, Wong SL, et al. Hospital volume and late survival after cancer surgery. Ann Surg 2007;245:777-83.

2. Hillner BE, Smith TJ, Desch CE. Hospital and physician volume or specialization and outcomes in cancer treatment: importance in quality of cancer care. J Clin Oncol 2000;18:2327-40.

3. Ahola R, Siiki A, Vasama K, et al. Effect of centralization on long-term survival after resection of pancreatic ductal adenocarcinoma. Br J Surg 2017;104:1532-8.

4. Gershman B, Meier SK, Jeffery MM, et al. Redefining and contextualizing the hospital volume-outcome relationship for robot-assisted radical prostatectomy: implications for centralization of care. J Urol 2017;198:92-9.

5. Joshi SS, Handorf ER, Sienko D, et al. Treatment facility volume and survival in patients with advanced prostate cancer. Eur Urol Oncol 2019. [Epub ahead of print].

6. Kim W, Wolff S, Ho V. Measuring the volume-outcome relation for complex hospital surgery. Appl Health Econ Health Policy 2016;14:453-64.

7. Leow JJ, Leong EK, Serrell EC, et al. Systematic review of the volume-outcome relationship for radical prostatectomy. Eur Urol Focus 2018;4:775-89.

8. Vrijens F, Stordeur S, Beirens K, et al. Effect of hospital volume on processes of care and 5 -year survival after breast cancer: a population-based study on 25000 women. Breast 2012;21:261-6.

9. Xia L, Pulido JE, Chelluri RR, et al. Hospital volume and outcomes of robot-assisted partial nephrectomy. BJU Int

Cite this article as: Kadow BT, Joshi SS, Kutikov A, Handorf E, Smaldone MC, Uzzo RG, Geynisman DM. The volume-outcome relationship in kidney cancer: is more really better? Ann Transl Med 2019;7(Suppl 8):S336. doi: 10.21037/ atm.2019.09.108
2018;121:900-7.

10. Luft HS, Bunker JP, Enthoven AC. Should operations be regionalized? The empirical relation between surgical volume and mortality. N Engl J Med 1979;301:1364-9.

11. Wasif N, Etzioni DA. Regionalization of complex cancer surgery: how, when, and why? JAMA Netw Open 2018;1:e184586.

12. Hsu RCJ, Salika T, Maw J, et al. Influence of hospital volume on nephrectomy mortality and complications: a systematic review and meta-analysis stratified by surgical type. BMJ Open 2017;7:e016833.

13. Becker A, Bianchi M, Hansen J, et al. Benefit in regionalization of care for patients treated with nephrectomy: a nationwide inpatient sample. World J Urol 2014;32:1511-21.

14. Joshi SS, Handorf EA, Zibelman M, et al. Treatment facility volume and survival in patients with metastatic renal cell carcinoma: a registry-based analysis. Eur Urol 2018;74:387-93.

15. Sun M, Bianchi M, Trinh QD, et al. Hospital volume is a determinant of postoperative complications, blood transfusion and length of stay after radical or partial nephrectomy. J Urol 2012;187:405-10.

16. Hsu RCJ, Barclay M, Loughran MA, et al. Impact of hospital nephrectomy volume on intermediate- to longterm survival in renal cell carcinoma. BJU Int 2019. [Epub ahead of print].

17. Waingankar N, Mallin K, Egleston BL, et al. Trends in regionalization of care and mortality for patients treated with radical cystectomy. Med Care 2019;57:728-33.

18. Stitzenberg KB, Sigurdson ER, Egleston BL, et al. Centralization of cancer surgery: implications for patient access to optimal care. J Clin Oncol 2009;27:4671-8. 